\title{
Implementação de ações educativas em saúde na sala de espera como estratégia para adesão de pacientes à vacinação em uma unidade básica de saúde
}

\author{
Sonia Francisca de Paula Monken ${ }^{1}$ \\ Jessica Lemos Pinheiro ${ }^{2}$
}

\begin{abstract}
RESUMO
A Unidade Básica de Saúde (UBS) é a porta de entrada aos usuários e também o centro de comunicação com toda a Rede de Atenção à Saúde. Na UBS são oferecidos diversos serviços em saúde, como por exemplo: O Programa Nacional de Imunizações, que contribui para a redução da morbidade e mortalidade. Ações de Educação em Saúde, são alternativas para empoderamento dos indivíduos sobre sua saúde. O objetivo deste trabalho foi analisar a contribuição de ações educativas para adesão à vacinação. Trata-se de estudo qualitativo, realizado em uma UBS. As atividades foram realizadas na sala de espera com orientações em saúde sobre a importância da vacinação. Após intervenções propostas foram orientados 201 usuários e 80 doses de vacinas foram aplicadas. $O$ presente trabalho apresentou resultados positivos, tanto para participação dos usuários nas atividades propostas quanto para a adesão dos orientados a realizarem à imunização.
\end{abstract}

PALAVRAS-CHAVE: Vacinação. Sala de espera. Unidade Básica de Saúde. Sistema Único de Saúde. Educação em saúde.

Implementation of educational actions in health in the waiting room as a strategy for patient adhesion to vaccination in a basic health unit

\footnotetext{
${ }^{1}$ Doutora.Universidade Nove de Julho, São Paulo, São Paulo, Brasil. Orcid: https://orcid.org/0000-0002-5063-8956. E-mail: sfmonken@hotmail.com.

${ }^{1}$ Mestranda em Sistemas de Gestão em Saúde. Universidade Nove de Julho, São Paulo, São Paulo, Brasil. Orcid: https://orcid.org/0000-0003-1634-5591. E-mail: jessica lemosp@ hotmail.com.
} 


\begin{abstract}
The Basic Health Unit (UBS) is the gateway for users and also the communication center with the entire Health Care Network. In the UBS various health services are offered, such as: The National Immunization Program, which contributes to the reduction of morbidity and mortality. Health Education Actions are alternatives for empowering individuals about their health. The objective of this work was to analyze the contribution of educational actions for adherence to vaccination. This is a qualitative study, carried out in a UBS. The activities were carried out in the waiting room with health guidelines on the importance of vaccination. After proposed interventions, 201 users were advised and 80 doses of vaccines were administered. The present study showed positive results, both for the participation of users in the proposed activities and for the adherence of those oriented to undergo immunization.
\end{abstract}

KEYWORDS: Vaccination. Waiting room. Basic Health Unit. Unified Health System. Health education.

Implementación de acciones educativas en salud en la sala de espera como estrategia de adhesión del paciente a la vacunación en una unidad básica de salud

\title{
RESUMEN
}

La Unidad Básica de Salud (UBS) es la puerta de entrada a los usuarios y también el centro de comunicación con toda la Red Asistencial. En la UBS se ofrecen diversos servicios de salud, tales como: El Programa Nacional de Inmunizaciones, que contribuye a la reducción de la morbilidad y la mortalidad. Las Acciones de Educación para la Salud son alternativas para empoderar a las personas sobre su salud. El objetivo de este trabajo fue analizar la contribución de las acciones educativas para la adherencia a la vacunación. Se trata de un estudio cualitativo, realizado en una UBS. Las actividades se realizaron en la sala de espera con pautas sanitarias sobre la importancia de la vacunación. Después de las intervenciones propuestas, se aconsejó a 201 usuarios y se administraron 80 dosis de vacunas. El presente estudio arrojó resultados positivos, tanto para la participación de los usuarios en las actividades propuestas como para la adherencia de las orientadas a la inmunización.

PALABRAS CLAVE: Vacunación. Sala de espera. Unidad Básica de Salud. Sistema Único de Salud. Educación para la salud.

$$
* * *
$$




\section{Introdução}

A Unidade Básica de Saúde (UBS) é a principal porta de entrada aos usuários e também o centro de comunicação com toda a Rede de Atenção à Saúde. Os serviços ofertados são todos gratuitos, uma vez que as unidades integram o Sistema Único de Saúde (SUS). Geralmente, os usuários frequentam unidades que estejam dentro do seu território de moradia, estudo ou trabalho. Na UBS são oferecidas consultas médicas e odontológicas, encaminhamento para especialidades, curativos, retirada de medicamentos, coleta de exames laboratoriais e vacinação.

Nos últimos anos têm se observado uma diminuição na adesão da imunização em pacientes adultos diante dos percalços rotineiros, como: horários divergentes (unidade e usuário) e falha de informação sobre o serviço ofertado aos usuários, além da somatização dos mitos e crenças que estão sendo criados nas mídias sociais e na cultura popular.

Com o intuito de aumentar a adesão dos pacientes e ofertar informações sobre o tema abordado, foi adotada como estratégia a implementação de ações educativas na sala de espera. A estratégia foi constituída visando atuar sobre o entendimento dos usuários, sendo realizada por meio de orientações com um jogo de perguntas e respostas, folders e cartazes explicativos sobre a importância da vacinação.

Assim, este relato técnico tem como objetivo responder a seguinte questão norteadora: "Qual a contribuição da educação em saúde na sala de espera para a adesão de pacientes à vacinação em uma UBS? ”. Desta forma, esse estudo está estruturado em quatro sessões, além desta breve introdução. A primeira sessão apresenta a revisão teórica-empírica do estudo, logo após, na segunda sessão será apresentado o método-empírico, seguido da sessão do levantamento e análise dos resultados, sendo finalizado com a quarta sessão, na qual será apontada as considerações finais dos autores. 


\section{Referencial Teorico}

O SUS é constituído por níveis de complexidade, divididos em: Atenção Primária à Saúde (APS), Atenção Secundária e Terciária. A APS emprega como método: orientar, responder e suprir as necessidades da população por meio da Estratégia Saúde da Família (ESF), priorizando ações de promoção, proteção e recuperação à saúde, de maneira integral e continuada. (GIOVANELLA et al., 2009; OLIVEIRA; PEREIRA, 2013).

No âmbito da UBS, ocorre o Programa Nacional de Imunizações (PNI), que contribui para a redução da morbidade e mortalidade por doenças transmissíveis. O PNI demostra ao longo dos anos um aumento na complexidade, uma vez que, em pouco tempo, introduziu-se diferentes vacinas no calendário de rotina e além disso, a cobertura vacinal do Brasil apresenta um desempenho comparável ao de países desenvolvidos (LIMA; PINTO, 2017; SATO, 2015; SILVA JUNIOR, 2013).

E mesmo com o País apresentando boas coberturas vacinais, os inquéritos apontam desigualdade entre os municípios, devido as diversas crises que influenciaram e ainda hoje influenciam na compreensão e aceitabilidade em relação a sua aplicação. Muitas variáveis estabelecem a população a terem opiniões diferentes, de caráter negativo, sobre a importância da vacinação, sendo essas questões demográficas, socioeconômicas, religiosas, científicas, políticas, falta de confiança devido experiências passadas e até mesmo por medo de agulha e de sentir dor (CHAVES; ROSS, 2014; DOMINGUES; TEIXEIRA, 2013; MIZUTA et al., 2019).

Visto a necessidade de contemplar as ações de promoção, proteção e recuperação à saúde, estratégias e ferramentas são utilizadas diariamente nos serviços de saúde, afim de desenvolver um atendimento humanizado e integral. Dentre as estratégias estão incluídas as atividades sobre Educação em Saúde, cujo é entendida como uma alternativa para transformação e empoderamento dos indivíduos sobre sua saúde. É importante ressaltar, que para desenvolver ações educativas em saúde é de extrema importância 
considerar o conhecimento popular e o contexto em qual estão inseridos (CHAVES; ROSS, 2014; MALLMANN et al., 2015).

Nesta circunstância, a sala de espera constitui-se em uma valiosa estratégia para efetivação de ações educativas, pois é um espaço amplo e de acesso a uma grande quantidade de usuários simultaneamente. As intervenções podem ser estruturadas a partir de diálogos entre acadêmicos, profissionais de saúde e usuários, sendo possível abordar diversos temas, desde necessidades locais ou até mesmo assuntos de importância nacional ou mundial, com o objetivo de promover estímulo ao autocuidado e aproximação entre comunidade e os serviços de saúde.(CALIXTO et al., 2018; ZAMBENEDETTI, 2012).

\section{Metodologia}

O referido trabalho foi realizado em uma UBS localizada na região Oeste de São Paulo pertencente a Coordenadoria Centro e a Supervisão Técnica de Saúde Santa Cecília. É uma unidade municipal, tendo como modelo misto de atuação (UBS Tradicional e Estratégia Saúde da Família ESF). Vale ressaltar, que no mesmo prédio, estão instalados programas do nível primário e secundário, sendo eles: Programa Acompanhantes de Idosos (PAI), Equipe Multidisciplinar de Atenção Domiciliar (EMAD) e Consultório na Rua. Além dos programas citados acima, também estão inseridos outros serviços de nível secundário, compondo-se por: Assistência Médica Ambulatorial (AMA) e Unidade de Referência à Saúde do Idoso (URSI).

Trata-se de estudo qualitativo, com atividades desenvolvidas pelos graduandos de Fisioterapia de uma universidade privada da grande São Paulo, que realizavam estágio em Saúde Coletiva na UBS referida, o grupo era composto por 8 alunos e um preceptor. No período de desenvolvimento da Sala de Espera, foram realizadas orientações em saúde por meio de um jogo de perguntas e repostas, folders e cartazes explicativos sobre a importância da vacinação. 
As orientações foram realizadas durante quatro dias não consecutivos, através de abordagens coletivas ou individuais com duração média de cinco minutos cada. Já o tempo total de atuação do grupo foi de cinco horas diárias. A presente metodologia foi realizada no período de abril a maio de 2019.

Para o levantamento do número de usuários participantes da sala de espera, foi desenvolvido em uma folha sulfite A4 um quadro com numerações de 0 a 100 e para cada usuário orientado um número era assinalado. O Cartão Nacional de Saúde (CNS) também foi utilizado para coleta de alguns dados, sendo eles: data de nascimento e sexo. Já para a verificação da adesão à vacinação, cada usuário era diretamente direcionado para sala de vacina e portando um cartão que continha a seguinte questão: Quais vacinas foram aplicadas? O cartão foi entregue ao final de cada orientação. Todos os pacientes foram acompanhados por um aluno e a questão do cartão preenchida pela equipe da enfermagem, ao final da imunização o cartão era entregue para o aluno acompanhante do paciente. Para melhor visualização, foi desenvolvido um fluxograma de atividades, com auxílio do software Bizagi (Figura 1).

FIGURA 1 - Fluxograma de atividades para implementação de ações educativas em saúde. São Paulo, Brasil, 2019.

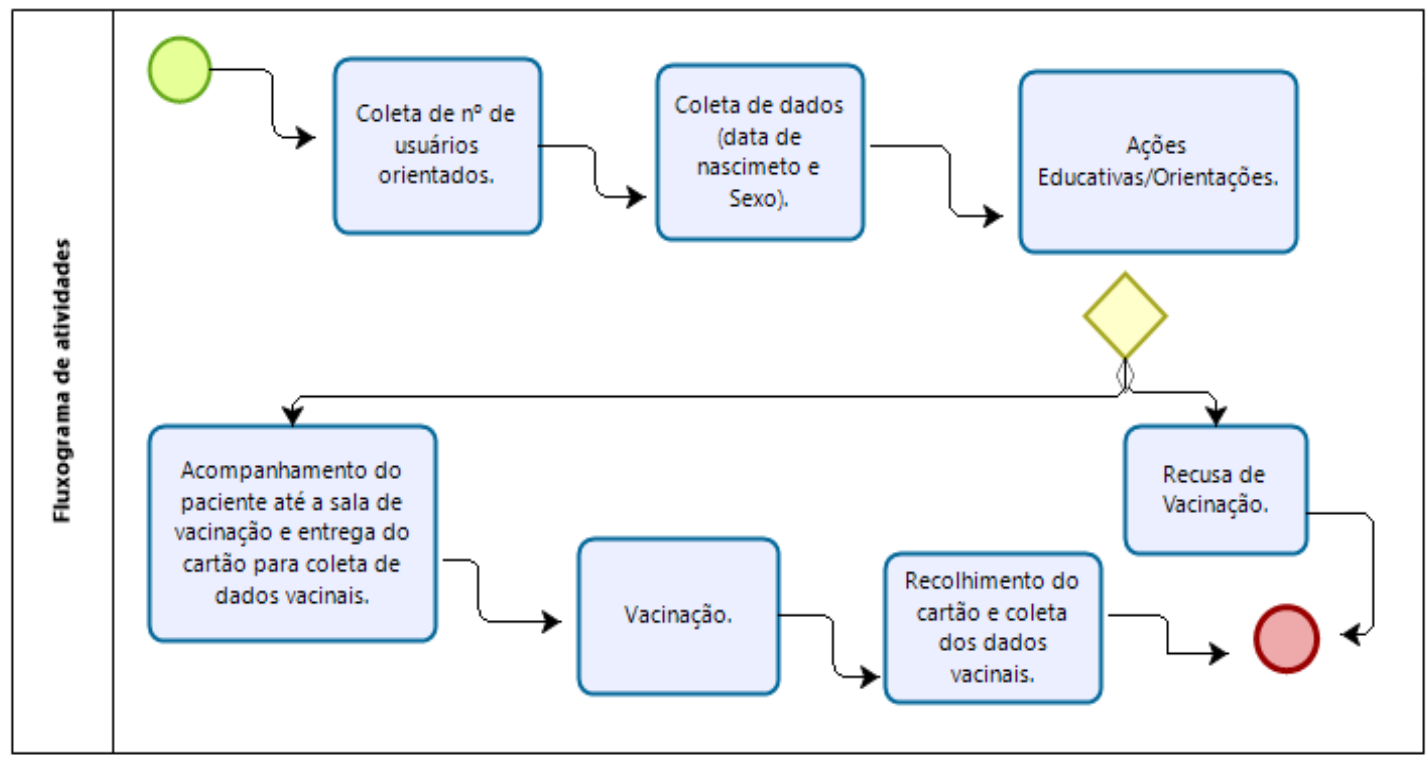

Fonte: Própria autora. 


\section{Resultados}

Diante a proposta de intervenção 201 usuários foram orientados, sendo $65 \%$ mulheres $(n=131)$ e $35 \%$ homens $(n=70)$. A média de idade foi de 59 anos e mediana de 63 anos. A menor idade foi de 19 anos e a maior 94 anos. No gráfico 1, podemos analisar os resultados quanto ao número de orientados por dia e o número de pacientes vacinados após as orientações de educação em saúde, ao total 64 pessoas foram vacinadas.

GRÁFICO 1 - Usuários orientados por dia e o número de vacinados após as orientações de Educação em Saúde. (n=201). São Paulo, Brasil, 2019.

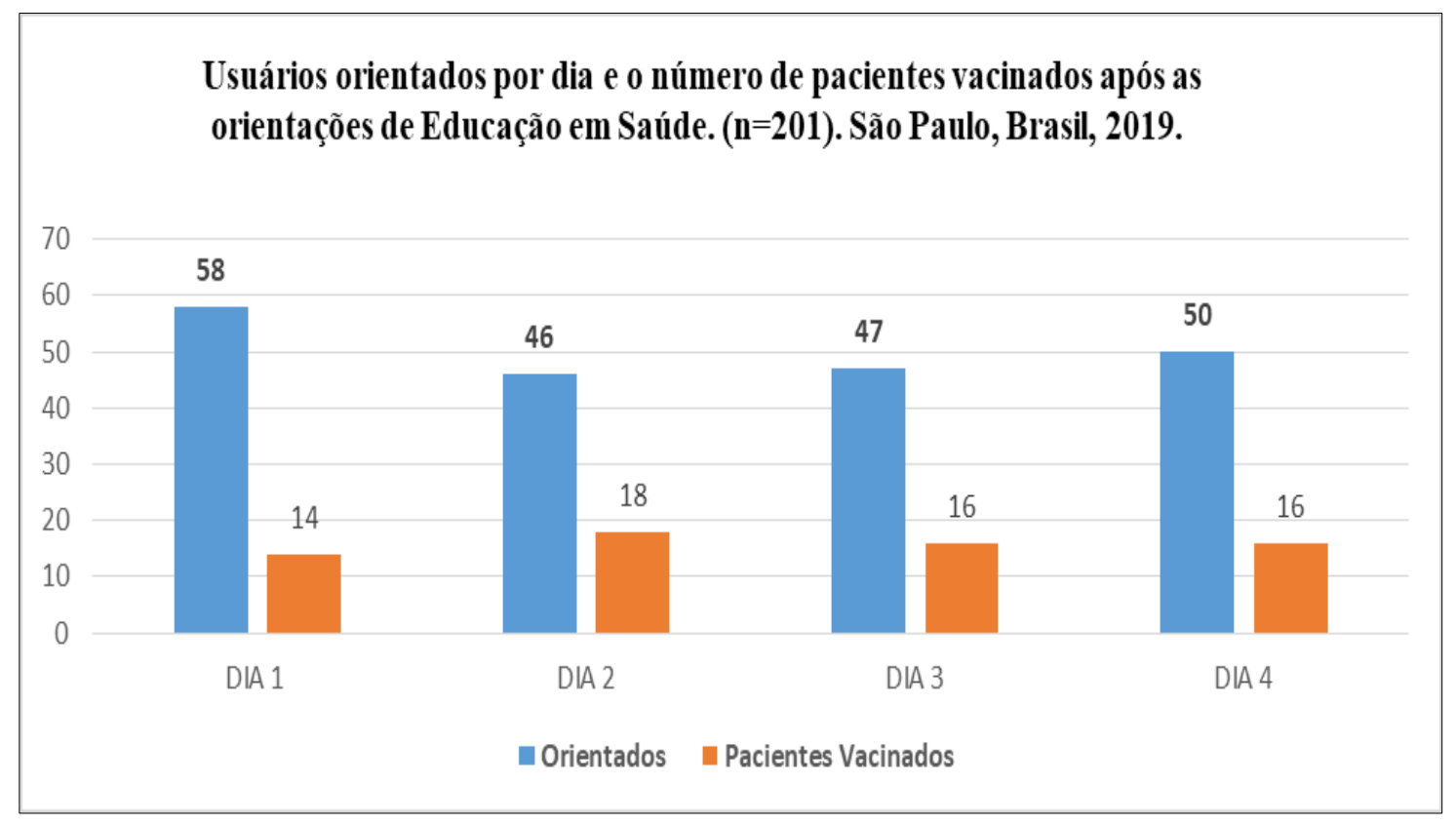

Fonte: Banco de dados

Em relação as vacinas, foram aplicadas 80 doses e as que mais se repetiram foram: Dupla Adulto (Difteria e Tétano - Dt), Febre Amarela, Hepatite B, Influenza e Tríplice Viral (Sarampo, Caxumba e Rubéola SCR). Os dados estão dispostos no gráfico 2 . 
GRÁFICO 2 - Doses e tipos de vacinas aplicadas, após orientação de Educação em Saúde. (n=201). São Paulo, Brasil, 2019.

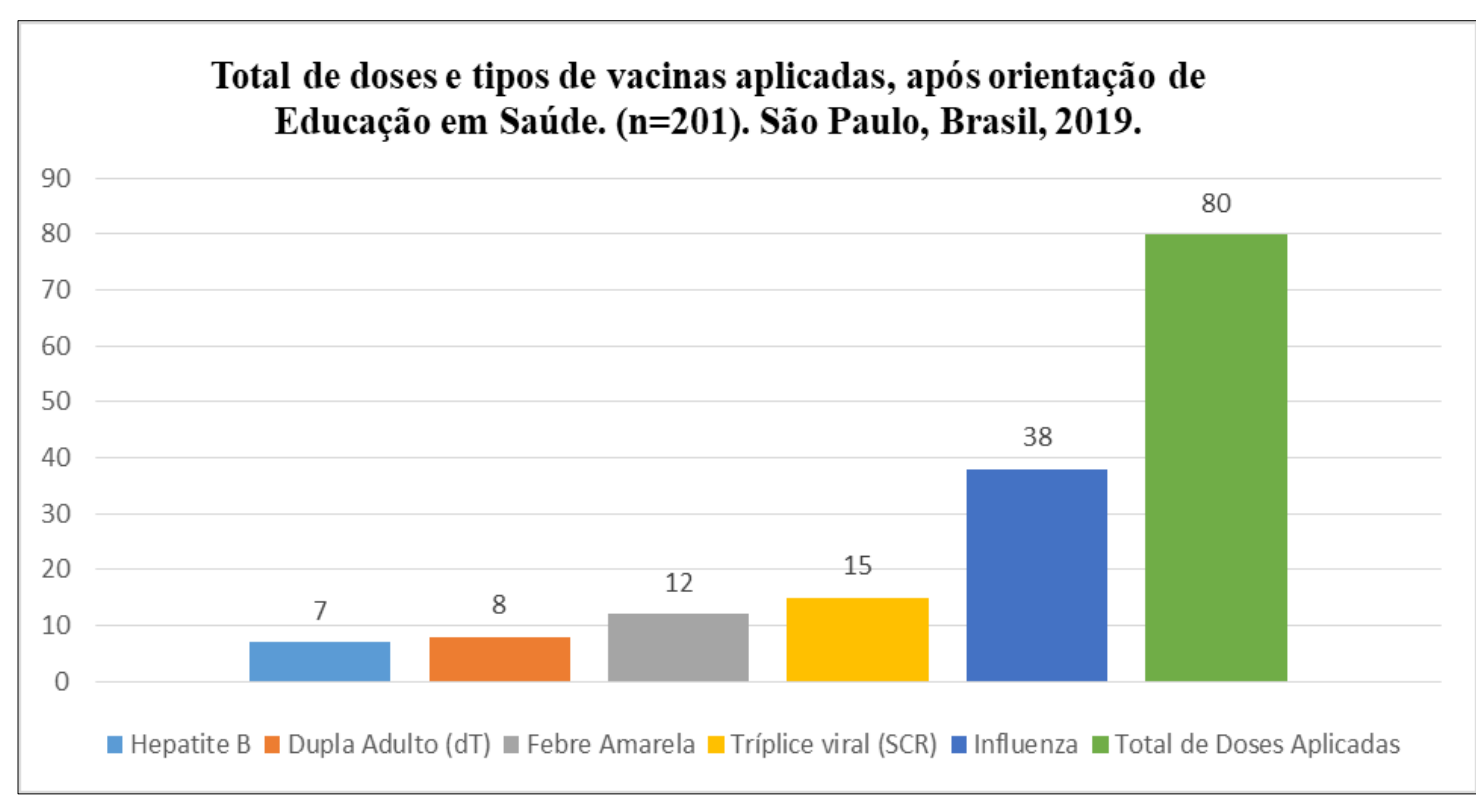

Fonte: Banco de dados

\section{Conclusão}

É importante salientar que anteriormente à condução do trabalho, ainda não havia sido realizada ações educativas em saúde quanto à importância da vacinação por meio da sala de espera. Desta forma, não foi possível mensurar em números resultados comparativos.

Contudo, podemos observar que o trabalho apresentou resultados positivos, tanto para participação dos usuários nas atividades propostas quanto para a adesão de $32 \%$ dos orientados a realizarem à imunização, demonstrando sua efetividade e viabilidade de implantação.

No presente estudo também foi possível reforçar o trabalho multiprofissional, no caso entre Fisioterapia e Enfermagem, além de salientar a importância da atuação da Fisioterapia com ações que promovam prevenção, promoção e educação à saúde na Atenção Primária.

Diante da comprovada contribuição do presente estudo para a literatura e a prática diária, considera-se relevante a realização de estudos complementares, visando - além de obtenção de parâmetros comparativos - 
delimitar a faixa etária em que a rejeição se observa maior, com intuito de se produzir ferramentas mais eficazes para o público alvo, derivando para uma otimização de recursos e aumento de adesão à vacinação.

\section{Referências}

CALIXTO, A. et al. Sala de espera: uma proposta para educação em saúde. Sinapse Múltipla, v. 7, n. 2, p. 188-195, 2018.

CHAVES, L. H. S.; ROSS, J. DE R. Perfil vacinal de adultos cadastrados em uma microárea da Estratégia Saúde da Família. Rev. enferm. UFPI, v. 3, n. 4, p. 4-9, 2014.

DOMINGUES, C. M. A. S.; TEIXEIRA, A. M. D.A S. Coberturas vacinais e doenças imunopreveníveis no Brasil no período 1982-2012: avanços e desafios do Programa Nacional de Imunizações. Epidemiologia e Serviços de Saúde, v. 22, n. 1, p. 9-27, mar. 2013.

GIOVANELLA, L. et al. Saúde da família: limites e possibilidades para uma abordagem integral de atenção primária à saúde no Brasil. Ciência \& Saúde Coletiva, v. 14, p. 783-794, 2009.

LIMA, A. A.; PINTO, E. DOS S. O contexto histórico da implantação do Programa Nacional de Imunização (PNI) e sua importância para o Sistema Único de Saúde (SUS). Scire Salutis, v. 7, n. 1, p. 53-62, 7 nov. 2017.

MALLMANN, D. G. et al. Health education as the main alternative to promote the health of the elderly. Ciência \&amp; Saúde Coletiva, v. 20, n. 6, p. 1763-1772, jun. 2015.

MIZUTA, A. H. et al. Percepções acerca da importância das vacinas e da recusa vacinal numa escola de medicina. Revista Paulista de Pediatria, v. 37, n. 1, p. 34-40, 2019.

OLIVEIRA, M. A. DE C.; PEREIRA, I. C. Atributos essenciais da Atenção Primária e a Estratégia Saúde da Família. Revista Brasileira de Enfermagem, v. 66, n. spe, p. 158-164, set. 2013. 
SATO, A. P. S. Programa Nacional de Imunização: Sistema Informatizado como opção a novos desafios. Revista de Saúde Pública, v. 49, p. 00-00, 1 jan. 2015.

SILVA JUNIOR, J. B. DA. 40 anos do Programa Nacional de Imunizações: uma conquista da Saúde Pública brasileira. Epidemiologia e Serviços de Saúde, v. 22, n. 1, p. 7-8, mar. 2013.

ZAMBENEDETTI, G. Sala de espera como estratégia de educação em saúde no campo da atenção às doenças sexualmente transmissíveis. Saúde e Sociedade, v. 21, n. 4, p. 1075-1086, dez. 2012. 